\title{
Qualche dubbio sul cadavere...
}

\author{
Luisa di Valvasone
}

PUBBLICATO: 21 GENNAIO 2020

\section{Quesito:}

Diversi lettori ci scrivono in merito alla voce cadavere. Alcuni si domandano quale sia la corretta etimologia della parola e se debba considerarsi un nome comune di cosa o di persona; altri invece ci chiedono se sia opportuno l'uso, prevalentemente giornalistico, di cadavere in funzione aggettivale in espressioni del tipo é stato ritrovato cadavere. Numerosi lettori inoltre segnalano l'impiego del termine riferito al corpo di un animale ritenendolo un uso scorretto e suggerendone la sostituzione con voci più appropriate come carcassa e carogna. Infine, qualcuno ci domanda se salma e cadavere possano considerarsi sinonimi.

\section{Qualche dubbio sul cadavere...}

ispondiamo con ordine alle molte domande.

\section{Quale etimologia?}

La parola cadavere è voce dotta dal latino cadāver, genitivo cadāveris, che ha avuto come esito originario nell'italiano antico la variante grafica cadavero, con metaplasmo ovvero, nella moderna linguistica, con un 'fenomeno morfologico per cui una parola passa da una declinazione o da una coniugazione ad altra' (Vocabolario Treccani online) in questo caso il passaggio alla classe dei nomi in -o (come fascio, sorcio, passero, cfr. LEI e Rohlfs I968 \$353); successivamente si è imposto nella forma oggi in uso cadavere che recupera la declinazione originaria (dall'accusativo cadavere $(m)$ ).

Il latino cadāver è presumibilmente connesso al verbo cădere 'cadere' (il GDLI rimanda a Isidoro di Siviglia, morto nel 636 d.C., che nelle sue Etymologiae scriveva: "cadaver nominatum a cadendo, quia iam stare non potest"; come non ricordare poi il dantesco e caddi come corpo morto cade), analogamente a quanto accade con il greco ptôma, -atos 'cadavere' (ma propriamente 'cosa caduta') derivato da pipto 'cadere' (DEI). Alcuni dizionari etimologici (l'Etimologico e Avviamento alla etimologia italiana di Giacomo Devoto, Firenze, Le Monnier, 1966) ipotizzano che si tratti di un adattamento di un participio perfetto attivo indoeuropeo in -wes di un possibile verbo *cadare 'esser caduto (definitivamente)' rispetto a cădere.

Tuttavia, fino all'Ottocento era in circolazione - e probabilmente lo è ancora oggi, dato che il suggerimento è giunto da un nostro lettore - una spiegazione piuttosto fantasiosa e certamente suggestiva dell'etimologia di cadavere: la ritroviamo ad esempio nel Dizionario enciclopedico delle scienze, lettere ed arti compilato da Antonio Bazzarini e pubblicato a Venezia nel i83o. Secondo questa teoria cadaver deriverebbe dalla contrazione delle prime tre sillabe della frase latina ca(ro) da(ta) ver(mibus), ovvero 'carne lasciata (in pasto) ai vermi'. È forse il caso di specificare che tale ipotesi, risalente "all'epoca prescientifica dell'etimologia" (LEI), veniva già smentita da molti dizionari ottocenteschi, e ancora da Pianigiani che nel 1907 scriveva nel suo Vocabolario etimologico: "altri ancora (per addurre un esempio di aberrazioni etimologiche) lo traggono dalle prime tre sillabe delle parole CA-ro, DA-ta, VER-mibus carne abbandonata ai vermi". Un caso analogo di etimologia popolare lo abbiamo visto, ad 
esempio, per la voce spa.

Per quanto riguarda la data di prima attestazione, il DELI indica prima del I364 per la variante ormai in disuso cadavero, nei Morali di San Gregorio Magno papa volgarizzati da Zanobi da Strada; l'attestazione, riportata nel GDLI e nel Vocabolario degli Accademici della Crusca - che già dalla prima edizione del I6I2 mette a lemma la variante cadavero -, fornisce l'indicazione etimologica poi ripresa dai dizionari: "Il cadávero si dice, secondo i gramatici, da cadére". Per cadavere invece il DELI indica il I623, in un testo di Giovan Battista Marino. La forma cadavere compare nella lessicografia della Crusca solo a partire dalla terza impressione, pubblicata nel I69I, all'interno delle voci andare (andare al morto) e portare (portare l'arme alla sepoltura), ma viene messa a lemma, accanto alla forma cadavero, solo nella quarta (I729-38) e nella quinta (I863-1923) impressione. Le attestazioni riportate nel GDLI anteriori al XVII secolo presentano tutte la variante cadavero, mentre quelle posteriori riportano sempre cadavere. Tuttavia, nel corpus TLIO possiamo già trovare 3 occorrenze della forma, coerente con il latino, cadavere in due testi, uno duecentesco di provenienza senese (I occorrenza, che il TLIO identifica come prima attestazione), l'altro trecentesco di area pisana (2 occorrenze):

Come Erittona fece i suoi incantesimi sul cadavere che avea trovato; e come pregò tutti i principi d'inferno perché l'anima chera uscita di quel corpo, vi tornasse con potenza di parlare, volendo Sesto conoscere la fine della battaglia. (Fatti di Cesare, Anonimo, XIII secolo, L. 6, cap. 20)

Anco è differente la Fede viva dalla morta, come l'animale vivo dal cadavere morto. Chè come il vivo animale si muove, ed ha vigore e valore, così la Fede viva si muove e vivifica l'anima: e per contrario lo cadavere, poniamo, che un poco palpiti, quando di fresco è morto, non è però quel movimento di vita, ma rimane tosto immobile e puzzolente (Esposizione del Simbolo degli Apostoli, Domenico Cavalca, I342, L.I, cap. II)

Nel testo di Cavalca troviamo inoltre unoccorrenza di cadaver riconducibile alla forma con -e, in quanto troncamento dovuto alla presenza della vocale iniziale e nella parola che segue:

Onde dice s. Bernardo: Che cosa è Fede senza amore, se non un cadaver esanime? Ben dunque onori Dio, o Cristiano, facendogli sacrificio si fetente! (Domenico Cavalca, Esposizione del Simbolo degli Apostoli, I342, L.I, cap. II)

Le occorrenze totali nel corpus TLIO sono dieci: 2 per la forma cadaver (ma una in un passo latino), 3 per il plurale cadaveri; 3, già viste, per cadavere e 2 per la variante con metaplasmo cadavero, in due testi trecenteschi fiorentini:

Né vivo poteo essere cacciato, ma morto per molte piaghe, il passaggio sopra il suo cadavero a loro (credo io ancora dopo la morte contra sua volontade) diede. (volgarizzamento del Défatti e detti degni di memoria della cittá di Roma e delle stranie genti di Valerio Massimo, Anonimo, I336, L. 4, cap. 6)

Nello odorato siamo avanzati dallo avoltoio, il quale, secondo i savi, sente il fiato del cadavero centinaia di miglia da lungi. (Agnolo Torini, Brieve collezzione della miseria della umana condizione, 1363-63, pt. 2, cap. I6)

Le occorrenze trovate sul corpus TLIO ci permettono, dunque, non solo di retrodatare cadavere, ma anche di constatare che l'alternanza tra le due varianti è presente in italiano dalle origini fino al XVII secolo (e in ambito letterario anche oltre: cadavero si trova, solo per fare alcuni esempi, nella poesia Carnevale di Carducci e nella Storia della letteratura italiana di De Sanctis), quando infine si impone la forma cadavere giunta fino ad oggi. 


\section{Nome comune di cosa o di persona (oppure di animale?)}

Nei testi scolastici di grammatica, in particolare delle scuole primarie e secondarie di primo grado, i nomi sono definiti attraverso categorie di opposti come: comuni e propri, astratti e concreti, individuali e collettivi. La distinzione che interessa ai nostri lettori, memori degli insegnamenti scolastici, è però quella che suddivide i nomi nelle tre categorie dette nomi di persona (Mario, dottoressa, nipote), nomi di animale (Yoghi, marmotta, sciame) e nomi di cosa (tavolo, carbonara, intelligenza). Questa schematizzazione mette sostanzialmente in opposizione i nomi che indicano qualcosa di animato (persone, animali) a quelli che qualificano ciò che è inanimato, sia esso astratto o concreto, malgrado la denominazione di cosa rimandi piuttosto a qualcosa di effettivo e materiale. Se possiamo facilmente affermare che cadavere è un nome comune, concreto e individuale, assai più complicato è stabilire se esso appartenga ai nomi di persona o ai nomi di cosa, senza considerare il fatto che, come vedremo, può essere riferito anche ad animali. Alcuni propendono per considerarlo nome di cosa, in quanto dopo la morte il corpo non è più animato, vivo; altri sostengono che, pur trattandosi di una cosa "inanimata", ogni corpo è stato comunque, in vita, identificabile con un essere senziente e quindi semanticamente collegato a un essere animato. Entrano in campo prevedibilmente le concezioni individuali, le nostre credenze e la nostra cultura. Davanti a ció, l'unica possibile considerazione è che questo tipo di classificazioni non ha alcuna influenza sull'aspetto morfologico delle parole e, sebbene simili categorie possano essere utili ai giovani studenti per meglio comprendere le basi della semantica e della grammatica, a un livello più profondo di analisi linguistica presentano diversi limiti. Tanto per fare un esempio: in molti si sono lamentati del fatto che nomi di fiori, alberi e piante siano considerati dalle grammatiche scolastiche come nomi di cosa, auspicando "l'istituzione"(!) della categoria "nomi di piante". L'idea può avere una sua logica, ma che dire allora dei nomi di luoghi geografici? E di tutte quelle parole che esprimono qualità e sentimenti tipici degli esseri umani? E i vocaboli che indicano parti del corpo animale? Per concludere dunque, intendere queste schematizzazioni in modo rigido rischia di impoverire la lingua stessa. Stabilire se cadavere appartenga ai nomi di persona, di animale o di cosa appare vano e finanche riduttivo delle variegate sfumature semantiche di cui il vocabolo è portatore.

\section{Sostantivo o aggettivo?}

Dal sostantivo cadavere derivano il diminutivo cadaverino, riferito al cadavere di un bambino, l'aggettivo cadaverico (prima attestazione I742, cfr. DELI) e l'ormai obsoleto cadaveroso, voce dotta dal latino cadaverosus. Alcuni lettori tuttavia fanno notare l'uso diffuso di cadavere come aggettivo, specialmente in costruzioni tipiche del linguaggio giornalistico del tipo l'uomo è stato trovato/ritrovato cadavere, in cui viene resa implicita la formula trovato/ritrovato (nello stato/nella condizione di) cadavere $\mathrm{e}$, in modo piuttosto naturale, cadavere assume il valore di predicativo del soggetto con il significato di 'morto, senza vita'. Probabilmente la scelta è guidata proprio dalla volontà di evitare la parola morto; l'uso dicadavere all'interno di lessici specialistici come quello della medicina legale, investigativo o dei romanzi gialli e polizieschi conferisce alla parola un aspetto tecnico dalla minore capacità evocativa rispetto a morto. Negli archivi di "Repubblica" e del "Corriere" troviamo numerosi esempi già a partire dal 1877 :

Ieri mattina lo hanno ritrovato cadavere sulla porta del suo ufficio, trafitto da trentacinque colpi di coltello molti dei quali alla gola ed al cuore. (Fatti diversi, "Corriere della Sera", 4/II/I877)

Un agricoltore israeliano è stato ritrovato cadavere ieri mattina a Shekef, un villaggio agricolo nei pressi della frontiera ovest della Cisgiordania, sessanta chilometri da Gerusalemme. Il corpo della vittima, Eliahu Cohen, è stato scoperto nelle prime ore della mattinata. Sul cadavere erano evidenti 
tracce di violenza, il cranio della vittima è stato sfondato da una sbarra di ferro. (Assassinato un colono israeliano, "La Repubblica", 2I/6/1988)

La misteriosa morte di una stella del cinema, trovata cadavere lungo la spiaggia del Lido di Venezia il giorno dopo la serata inaugurale della Mostra del Cinema, è al centro del nuovo romanzo di Antonella Boralevi "Chiedi alla notte". (Nel cuore della notte, Boralevi racconta una morte a Venezia, "La Repubblica.it", 19/5/2019)

L'uso è evidentemente diffuso da tempo nella lingua della stampa, sebbene Arrigo Accornero, nel suo Manuale di linguaggio giornalistico del I976, lo segnalasse come errore da evitare:

Cadavere - è un sostantivo e non un aggettivo (mentre morto può essere l'uno o l'altro): per questo non si può dire "l'uomo è stato trovato cadavere"; molto meglio "il cadavere di un uomo è stato trovato". Così anche si eviti di dire "l'uomo è giunto cadavere all'ospedale"; meglio: "l'uomo è morto durante il trasporto all'ospedale" (Arrigo Accornero, Manuale di linguaggio giornalistico, Milano, ETAS libri, 1976)

Nei dizionari sincronici (GRADIT, Sabatini-Coletti 2008, Garzanti 2017, Devoto-Oli 2019, Zingarelli 2020, Vocabolario Treccani online), nel Tommaseo-Bellini e nella quinta impressione del Vocabolario degli Accademici della Crusca cadavere è sempre indicato come sostantivo maschile. Ciò nonostante in alcuni esempi d'uso riportati nelle voci lessicografiche troviamo espressioni come trovare qualcuno già cadavere (GRADIT), lo trovó già cadavere (Vocabolario Treccani online), arrivó all'ospedale già cadavere (Devoto-Oli 2019). L'unico dizionario che pone a lemma cadavere con valore aggettivale nel significato di 'morto' è il GDLI, che lo segnala però come "antico" e riporta un'attestazione dal trattato Del ghiaccio e della coagulazione (I edizione I680) del gesuita Daniello Bartoli: si ardi a definire, quello [sangue] delle arterie essere animato e vivo, l'altro delle vene, morto e cadavero. Nell'italiano letterario tuttavia l'uso è proseguito ben oltre il XVII secolo, come dimostrano le attestazioni successive al Bartoli trovate in rete e in banche dati come la BIZ:

Ma non appena l'ebbi ghermita, mi si disfece tra le braccia; il capo ricadde, le carni si sciolsero in putridume; e rimasi muto scerpelloni, a bocca aperta, più cadavere che colei nol fosse sembrato poco prima; mentre l'uscio si spalancava per gli sforzi replicati ed un fragore ed un chiarore straordinario irrompevano nelle tenebre e nel silenzio del tempio. (Vittorio Imbriani, Merope IV, r867)

Chi volge a fuga il piede, / uno è cadavere, / uno è ferito, quest'altro supplica / d'aver salva la vita. (Euripide, Le Tragedie. I poeti greci tradotti da Ettore Romagnoli, volume V: Elettra, Oreste, traduzione di Ettore Romagnoli, Bologna, Zanichelli, 1930)

"Per ora le posso dire picca, devo taliàre altre cose. È certo che sono morti sparati. Lui con un colpo alla tempia, lei con un colpo al cuore. La ferita della femmina non si vedeva perché ci stava sopra la mano di lui. Un'esecuzione in piena regola, mentre dormivano".

"Dentro la grotta?"

"Non credo, penso che siano stati portati lì già cadaveri e quindi ricomposti, nudi com'erano". (Andrea Camilleri, Il cane di terracotta, Palermo, Sellerio Editore, 1996)

Il fenomeno non è estraneo alla nostra lingua; come scrive Serianni nella sua Grammatica italiana (Serianni 1989 cap.V, L'aggettivo e il nome): "la grande affinità di forme e impieghi esistente tra aggettivo e nome fa si che le due categorie spesso assumano l'una le funzioni dell'altra" e, anche se l'impiego del nome come aggettivo è meno comune rispetto al caso inverso, non mancano esempi letterari, come quelli riportati da Serianni: "così briciola com'era" (Nievo, Le confessioni di un italiano), "tutto quell'esibito bollore di menti bambine che si sentivano crescere" (Bufalino, Diceria dell'untore), "Com’e alto il dolore. / L'amore, com’e bestia" (Caproni, Senza esclamativi). Si guardi tra gli altri la 
citazione tratta dal romanzo Demetrio Pianelli di Emilio De Marchi (ı89o): "oltre a essere per sua natura invidiosa e vespa“, dove il sostantivo vespa assume il valore di aggettivo nel significato di 'cattiva, pungente come una vespa', analogamente a quanto accade nei casi visti finora di cadavere. Se però in ambito letterario la funzione aggettivale di cadavere può rappresentare una scelta di stile, nel linguaggio giornalistico, per quanto già in passato sia stata sconsigliata, pare invece una cristallizzazione, in particolare nella formula trovato/ritrovato cadavere, che richiederebbe, almeno, un uso più circoscritto.

\section{Si può usare cadavere riferendosi al corpo morto di un animale?}

I dizionari novecenteschi (il Dizionario della lingua e della civiltá italiana contemporanea di Emidio De Felice e Aldo Duro del 1974, il Dizionario della lingua italiana di Palazzi-Folena del 1992, lo Zingarelli 1994) e i sincronici contemporanei (Sabatini-Coletti 2008, Devoto-Oli 2019, lo Zingarelli 2020, Vocabolario Treccani online) concordano nel definire cadavere unicamente il 'corpo umano dopo la morte'. Rappresentano un'eccezione il GRADIT ('nell'uso corrente anche carogna di animale') e il Garzanti 2017 che indica l'accezione animale come non comune.

Risalendo negli anni scopriamo che i dizionari ottocenteschi (come il Tommaseo-Bellini, il Vocabolario della lingua italiana di Antonio Sergent del 1870 e la quinta impressione del Vocabolario degli Accademici della Crusca) non applicano una distinzione cosi categorica; i più specificano che cadavere indica un generico corpo morto ma 'detto piu specialmente del corpo umano', anche se nel Vocabolario italiano della lingua parlata di Rigutini-Fanfani pubblicato nel 1875 possiamo leggere: "il corpo dell'uomo dopo morto: delle bestie oggi non si dice". Le prime quattro edizioni del Vocabolario degli Accademici della Crusca (I6I2, I623, I69I, I729-38) invece non applicano alcuna distinzione. Infine il TLIO, dizionario storico dellitaliano delle origini, evidenzia distinguendole entrambe le accezioni, umana e animale, e riporta per la seconda due esempi d'uso: uno nell'opera di Cavalca del I342 e l'altro nel testo fiorentino di Agnolo Torini datato 1363-74, entrambi già visti a proposito dell'etimologia di cadavere.

Dunque, è certo che in origine il termine cadavere potesse egualmente indicare il corpo senza vita di un uomo come quello di un animale. Nel dizionario storico GDLI non viene fatta alcuna distinzione e tra gli esempi d'uso riportati troviamo due attestazioni di cadavere riferito ad animali (ma anche alle piante!) tratte da Galileo, la prima, e dallo studioso settecentesco Tommaso Perelli, la seconda:

Infragnerò dunque e strapiacerò l'istesso scorpione sopra le ferite, onde il veleno risorbito dal proprio cadavero lasci me libero e sano.

Dee intendersi... de' cadaveri delle piante, de' rettili, e soprattutto degl'insetti.

L'alternanza tra i due usi, tuttavia, sembra essersi interrotta a partire dal XIX secolo e, nonostante le eccezioni rappresentate da GRADIT e Garzanti 20I7, le indicazioni lessicografiche contemporanee sono chiare e concordi in merito: attualmente l'impiego di cadavere riferito al corpo animale è discutibile. Ma si sa, non sempre gli strumenti lessicografici riflettono le reali tendenze dei parlanti e l'italiano di oggi è ricco di esempi d’uso:

Il cadavere di un tonno, sfuggito dalle mani dell'addetto, scivolava dolcemente sul pavimento, tentando un'impossibile fuga. (Stefano Benni, Spiriti, Milano, Feltrinelli Editore, 2002)

Gli episodi riportati erano concentrati soprattutto nel quartiere di Cambian Park. Poco dopo cominciarono ad essere ritrovati cadaveri di gatti. (Usa: uccise 21 gatti, condannato a 16 anni di prigione, "Repubblica.it", sezione Esteri, 15/7/2017) 
Non mancano le lamentele per questo uso impreciso di cadavere; oltre ad alcuni nostri utenti, troviamo un esempio online nella lettera di un lettore del "Corriere", medico legale, che rimprovera il giornale per l'uso di cadaveri riferito a uno sfortunato stormo di tortore (citando peraltro l'etimologia che vorrebbe derivare la parola da "caro data vermibus") e al quale Sergio Romano risponde che "usata per gli uccelli la parola è un segno di sensibilità per gli animali che piacerà, se non ai medici legali, ai veterinari".

L'italiano contemporaneo dispone di due vocaboli per descrivere il corpo di un animale morto che molti dei nostri lettori ritengono più corretti di cadavere: carcassa e carogna. Riprendendo le definizioni del Devoto-Oli 2019, il primo termine indica propriamente 'l'insieme delle ossa che formano la cavità toracica degli animali' e per estensione 'lo scheletro di un animale', mentre carogna, presente nella nostra lingua già dal XIII secolo, indica propriamente il 'corpo di animale morto in putrefazione'. Per quest'ultima Serianni (Il primo Ottocento, Bologna, Il Mulino, I989, p. 45) riporta il caso del purista Antonio Cesari che, tra il XVIII e il XIX secolo, fu accusato di aver usato carogna come sinonimo di 'cadavere umano'; nonostante la presenza sul GDLI di alcuni esempi di tale uso duequattrocenteschi, Cesari smenti fermamente e defini l'accusa una calunnia. Entrambe le voci carcassa e carogna hanno poi usi estesi e figurati dalla connotazione prevalentemente spregiativa: carcassa è detto anche di 'persona dal corpo sfatto e malandato' e di oggetti, specialmente mezzi di trasporto, 'in pessime condizioni'; carogna è anche una 'bestia sfiancata e malridotta' o, più comunemente, una 'persona subdola e maligna, capace d'ogni perfidia'. È naturale dunque che i due termini possano essere percepiti come poco rispettosi nei confronti degli animali; optare per l'uso di cadavere (uso che, come abbiamo visto, non è affatto estraneo all'italiano fin dalle sue origini) sembra sottintendere la personificazione dell'animale - non solo nel caso di animali domestici - e può essere segnale di affetto e rispetto verso esseri viventi che, se anche soltanto in alcuni casi fanno parte della nostra quotidianità, sono da sempre nostri fondamentali "coinquilini" in questo pianeta. Le occorrenze trovate online dimostrano inoltre una prevalenza d'uso da parte della stampa, come nel caso dell'articolo pubblicato sulla "Repubblica" segnalatoci da un nostro lettore. Ne riportiamo alcuni passi, in cui l'uso di cadavere, alternato a carcassa, è visibilmente uno strumento di personificazione del piccolo di delfino, protagonista della triste vicenda, volto a impressionare e coinvolgere emotivamente i lettori:

Un cucciolo di delfino senza vita in mezzo al mare. Ma non è stato lasciato solo. Una coppia di femmine, fino all'arrivo di un velista nelle acque a largo di Viareggio, ha scortato il cadavere del piccolo tra le onde, come se si trattasse di una veglia. [...] Dopo 40 minuti di osservazione sul comportamento degli individui adulti, l'equipaggio della motovedetta è riuscito a recuperare la carcassa del piccolo delfino per portarla in porto per le necessarie analisi necroscopiche [...]. Il cadavere del cucciolo è stato consegnato ai ricercatori dell'Università di Siena e le analisi verranno svolte insieme all'Arpat e all'Istituto zooprofilattico di Pisa. Oltre al piccolo esemplare di Viareggio, un altro delfino tursiope è stato trovato in stato di decomposizione a Capoliveri, all'isola d'Elba. (Claudio Cucciatti, Ancora delfini morti in Toscana: il cadavere del cucciolo viene "vegliato" da due fermmine, "La Repubblica", 31/7/2019)

\section{Cadavere e salma sono sinonimi?}

Oltre al primo significato di 'carico, peso' e a quello di 'antica unità di misura', la parola salma ha altri due significati: quello, poetico-letterario e attestato a partire dal I294 (DELI), di 'corpo umano, la parte fisica dell'uomo, in quanto carico corporeo dell'anima' (Vocabolario Treccani online); e quello, comune e attestato dal XVII secolo, di 'spoglie mortali, cadavere'. Secondo il GRADIT vi è differenza rispetto alla frequenza d'uso: cadavere (e così carogna) appartiene al lessico fondamentale (FO; "sono cosi marcati i vocaboli di altissima frequenza, le cui occorrenze costituiscono circa il $90 \%$ delle occorrenze lessicali nell'insieme di tutti i testi scritti o discorsi parlati”; GRADIT, Introduzione, §II), mentre salma (e cosi carcassa) è marcata sul dizionario con la sigla CO ("sono cosi marcati i vocaboli 
che sono usati e compresi indipendentemente dalla professione o mestiere che si esercita o dalla collocazione regionale e che sono generalmente noti a chiunque abbia un livello mediosuperiore di istruzione"), cioè fa parte del lessico comune e dunque è meno frequente rispetto a cadavere. Consapevoli che la sinonimia perfetta è un concetto teorico, ciò che interessa ai nostri lettori è capire se esistano contesti in cui le due voci possano essere usate indistintamente. Guardando ai dizionari, possiamo verificare che la definizione di salma in quanto 'corpo umano morto' è sempre corredata dalla specificazione di 'oggetto di onori funebri' (GRADIT) usato 'nel linguaggio devoto in riferimento all'aspetto sacro delle esequie' (Devoto-Oli 2org), ovvero un cadavere 'già composto per la sepoltura' (Vocabolario Treccani online). Dunque, se è vero che sia cadavere sia salma indicano un corpo umano senza vita, il secondo termine ha però rispetto al primo 'connotazioni di elevatezza, di affetto, di devozione' (Sabatini-Coletti 2008). Va detto, tuttavia, che anche gli addetti delle onoranze funebri usano spesso salma anche prima di comporre il corpo del defunto; perlopiù la scelta è dettata dalla volontà di evitare termini come corpo, morto e, appunto, cadavere, percepiti come troppo diretti e "crudi".

Diverse sono le occorrenze trovate in rete nelle quali salma è usato come evidente sinonimo di cadavere (a cui si aggiungono altri sinonimi come corpo e corpo senza vita); alcuni esempi:

Il cadavere di una donna ucraina di 57 anni è stata trovato [sic] in via Casilina ${ }^{7} 75^{2}$, nella tarda serata di ieri intorno alle 22. Da una prima ricostruzione sembra si tratti di un investimento ad opera di un auto [sic] o di un motociclo dato che la salma è stata trovata vicino alle strisce pedonali. (Roma, donna trovata morta su un marciapiede: forse è stata investita, "il Messaggero.it", 15/5/2019)

La salma è stata recuperata nella mattina di sabato ir novembre dai Vigili del fuoco, dopo una notte in cui i carabinieri hanno sorvegliato l'area affinché non venisse inquinata l'area. In attesa di accertamenti medici più dettagliati, da una prima analisi del cadavere si desume che la donna era di carnagione chiara e che la morte risale a circa due mesi fa. La salma è stata ritrovata in una zona in cui non risultano denunce di persone scomparse corrispondenti al profilo finora tracciato della donna defunta. (Torino, ritrovato cadavere di una donna: la morte risale a circa due mesi fa, "Fanpage.it", II/II/2017)

Il corpo della piccola Sara è stato individuato da un bagnante ieri mattina nello stesso tratto di mare dove la ragazza è affogata. Pochi minuti e una vedetta della Capitaneria di porto, coadiuvata da una pattuglia della polizia municipale, ha provveduto a portare a riva la salma. (Trovata la salma della dodicenne affogata domenica, "SenigalliaNotizie", 22/7/2003)

Ancora una volta è perlopiù nel linguaggio giornalistico che troviamo la maggior parte delle occorrenze. In nessuno dei casi riportati si parla di corpi già composti per le esequie, ma l'alternanza è spesso utile per evitare casi di ripetizione. Per quanto si tratti di un uso semanticamente impreciso, in nessun caso si corre il rischio di ambiguità e incomprensioni, e il contesto quasi sempre chiarisce il significato. Certo, potrà far storcere il naso trovare salma usato in riferimento a un cane, specialmente a coloro che già si erano lamentati a causa dell'impiego di cadavere nel caso di animali:

Ora al Cemivet stanno cercando di capire se la salma o almeno le ceneri del pastore belga potranno essere riportate in Italia, in base alle leggi in materia sanitaria vigenti nel Paese nel quale stava operando. (Autopsia sulla salma del "cane eroe", "Il Tirreno", 6/4/2018)

La situazione cambia naturalmente quando entriamo nell'ambito giuridico e in particolare all'interno delle normative che regolano le attività della polizia mortuaria e in generale tutte le attività funebri, dove la distinzione tra salma e cadavere assume significato tecnico e valore legale ed è pertanto strettamente vincolata alle disposizioni del legislatore. Poiché la discussione è ancora in corso, ci 
limitiamo in questa sede a riportare le definizioni presenti nel testo della proposta di legge presentata il 6 settembre 2018 riguardo alla "Disciplina delle attività funerarie, della cremazione e della conservazione o dispersione delle ceneri”:

Ai fini della presente legge: a) per «salma» si intende il corpo umano rimasto privo delle funzioni vitali fino a ventiquattro ore dalla constatazione del decesso o prima dell'accertamento della morte; b) per «cadavere» si intende la salma, dopo le ventiquattro ore dalla constatazione del decesso o dopo l'accertamento della morte secondo quanto previsto dalla legislazione vigente. (proposta di legge Ir43, Disciplina delle attivitá funerarie, della cremazione e della conservazione o dispersione delle ceneri, titolo I, art. 2 Definizioni)

\section{Cita come:}

Luisa di Valvasone, Qualche dubbio sul cadavere... , "Italiano digitale", 2020, XII, 2020/1 (gennaiomarzo)

DOI: $10.35948 / 2532-9006 / 2020.3318$

Copyright 2020 Accademia della Crusca

Pubblicato con licenza creative commons CC BY-NC-ND 